\title{
Avanzando en la lucha contra la tuberculosis en Chile
}

\author{
CARLOS PEÑA M.* y VICTORINO FARGA C.**
}

\section{Moving forward in the fight against tuberculosis in Chile}

In 1973 Chile started a National Tuberculosis Control Programme based in the use of sputum smear for tuberculosis finding and proper treatment. Programme activities lead the country to achieve the threshold of tuberculosis elimination in year 2000. Since then the usual annual 7\% decrease in the incidence of tuberculosis has leveled off. Between years 2009-2011 tuberculosis rate remained stationary in around 13.1 per 100.000 inhabitants. Such epidemiologic regression was related to a decrease in bacteriological case finding on primary health care and the existence of risk groups like the elderly, prisoners, migrants and HIV infected. The Metropolitan Region of Santiago represents $40 \%$ of the total incidence of tuberculosis of the country and has a higher proportion of high risk groups. We recommend to increase the tuberculosis detection rate focused in high risk groups, to increase preventive therapy of latent tuberculosis in HIV infected and close child contacts and improve treatment success. In year 2012 we have achieved a lower tuberculosis incidence rate of 12.6 per 100.000 inhabitants due to an increase in sputum smear detection. It is necessary to maintain this measures for the continuous progress in the control of tuberculosis.

Key words: Control of Tuberculosis Programme; Tuberculosis high risk groups; latent tuberculosis; preventive tuberculosis therapy.

\section{Resumen}

Chile inició un programa de actividades de pesquisa bacteriológica y tratamiento de la tuberculosis a través de toda la red de salud nacional en 1973. Producto de esta iniciativa el país alcanzó el umbral de inicio de la eliminación de la enfermedad el año 2000. Desde entonces se ha enlentecido la velocidad de reducción de la incidencia de la enfermedad desde 7\% anual hasta detenerse el año 2009. Entre el año 2009 y 2011 la incidencia se ha mantenido estacionaria con alrededor de 13,1 casos $x 100.000$ habitantes. Se han identificado algunos factores que han contribuido a esta detención epidemiológica, como por ejemplo la reducción de la pesquisa de los casos pulmonares bacilíferos, especialmente a nivel de atención primaria. También se ha encontrado una mayor concentración de casos entre población vulnerable constituida por grupos de riesgo como adultos mayores, personas privadas de libertad, migrantes e infectados por VIH. La Región Metropolitana aporta el $40 \%$ de los casos de tuberculosis y la proporción de población de riesgo de enfermar es mayor que en el resto del país. En el presente artículo se proponen estrategias para revertir esta situación. Las principales medidas son: incrementar la detección de casos en todos los niveles de salud con intensificación en los grupos de riesgo; aumentar las medidas preventivas con quimioprofilaxis en los infectados VIH y contactos infantiles y optimizar los resultados del tratamiento para interrumpir la cadena de transmisión de la enfermedad. Aún hay esperanzas. El año 2012 la incidencia se redujo a 12,6 x 100.000 y se incrementó la pesquisa baciloscópica después de varios años en que había disminuido. Mantener estas medidas se hace necesario para seguir avanzando en el control sanitario de la tuberculosis.

Palabras clave: Programa Control de Tuberculosis; grupos de alto riesgo; tuberculosis latente; tratamiento preventivo antituberculosis.

\footnotetext{
* Jefe Programa de Control y Eliminación de la Tuberculosis, Servicio de Salud Metropolitano Central.

** Asesor del Programa de Control y Eliminación de la Tuberculosis, Ministerio de Salud de Chile.
} 


\section{Situación actual de la tuberculosis}

La tuberculosis (TBC) fue declarada una Emergencia Global por la OMS hace dos décadas. En el año 2011 se reportaron 8.700 .000 de casos nuevos y murieron por esta causa 1.400 .000 personas ${ }^{1}$. En Chile se está dando una lucha continua contra la enfermedad a través de un Programa Nacional de Control de la Tuberculosis iniciado en 1973. Las actividades del programa están centradas en la detección de los casos que transmiten la enfermedad utilizando una estrategia de localización de casos o pesquisa con baciloscopía en los sintomáticos respiratorios que consultan espontáneamente en todos los Servicios de Salud del país y tratando en forma gratuita y bajo supervisión directa de personal sanitario entrenado todos los casos diagnosticados ${ }^{2}$. Esto permitió reducir progresivamente la incidencia de la TBC en casi 7\% anualmente durante la década de los 90. Para el año 2000 el país presentaba una tasa de incidencia de TBC de todas las localizaciones menor a $20 \times 100.000$ habitantes, ingresando a la etapa epidemiológica de inicio del umbral de eliminación. Sin embargo, desde esa fecha el ritmo de reducción anual ha sido notablemente menor y desde el año 2009 la tendencia de la enfermedad se mantiene estacionaria en un promedio de $13,1 \times 100.000$ habitantes $^{3}$ (Figura 1).

Esta situación se ha atribuido en buena parte a la menor intensidad de la pesquisa baciloscópica de los casos de TBC pulmonar bacilífera, especialmente a nivel de Atención Primaria de Salud, lo que ha mantenido la transmisión de la enfermedad en la comunidad (Figura 2). También se ha vinculado esta situación a la presencia de la enfermedad en grupos poblacionales más vulnerables como son los adultos mayores, personas privadas de libertad (PPL), migrantes, co-infectados por VIH y otros en los cuales la enfermedad es más frecuente. La proporción de estos grupos se mantiene estable desde las primeras evaluaciones realizadas en el país el año 2000 hasta 2012 (Tabla 1).

Se aprecia un comportamiento variable de la incidencia de la TBC en los distintos Servicios de Salud del país, ya que la mitad de ellos (15 de
Figura 1. Tasas de incidencia de tuberculosis de todas las localizaciones. Chile. Años 1989-2012. Fuente: PROCET.
Figura 2. Índice de pesquisa. Chile. Año 2012. Fuente: PROCET
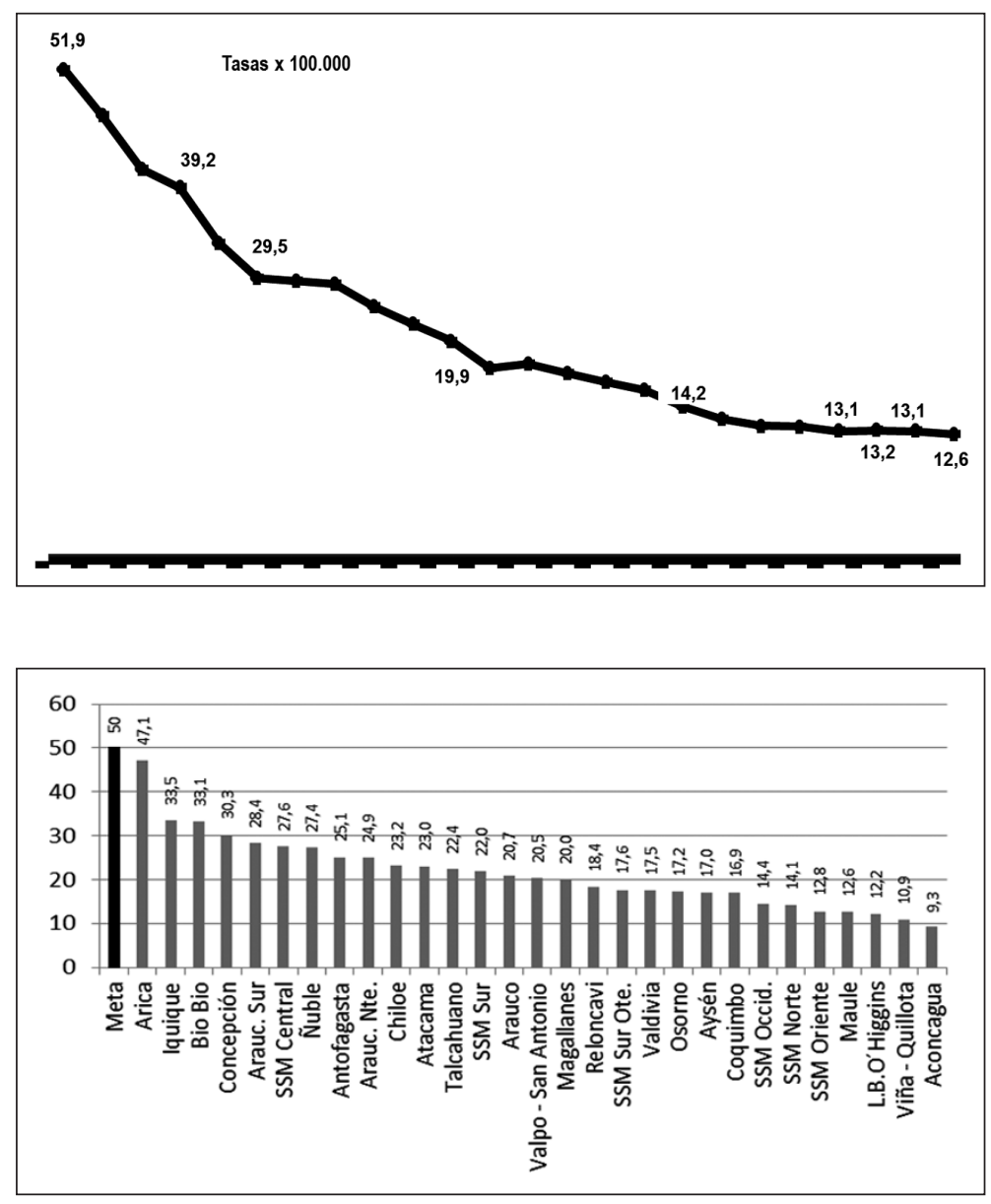
29) tiene una tasa de tuberculosis sobre el promedio nacional. Estos servicios tienen un promedio de incidencia de 19,1 x 100.000, que es la situación epidemiológica que presentaba el país hace más de una década (Tabla 2).

Los resultados del tratamiento de la tuberculosis en la cohorte de pulmonares bacilíferos del año 2011 reportaron un éxito de sólo $82,1 \%$. Estos resultados se ven afectados por la alta proporción de fallecidos $(8,5 \%)$ y abandonos $(6,5 \%)$, (Figura 3).

\section{Importancia de la Región Metropolitana}

La Región Metropolitana (RM) posee una población estimada de 7.007.620 habitantes el año 2012. Está constituida por 52 comunas de 6 provincias (Santiago, Maipo, Melipilla, Cordillera, Talagante y Chacabuco).

La situación de la TBC en la RM se mantiene estacionaria entre el año 20082010 y luego presenta una ligera declinación. La tasa promedio de TBC de todas las localizaciones entre el período 20062012 es de 14,8 x 100.000 habitantes ${ }^{4}$. Sin embargo, la tasa de TBC pulmonar bacilífera del año 2012 es similar a la del año 2007 (tasa 6,6 x 100.000), lo que indica que se mantienen en circulación los vectores de la transmisión (Figura 4).

La carga de enfermedad de la región
Tabla 1. Proporción de grupos vulnerables en la incidencia de tuberculosis de Chile

\begin{tabular}{|lccc|}
\hline Grupos vulnerables & $\mathbf{2 0 0 5 - 2 0 0 7}$ & $\mathbf{2 0 1 1}$ & $\mathbf{2 0 1 2}$ \\
\hline Privados de libertad (\%) & 6,4 & 3 & 4,3 \\
\hline Migrantes (\%) & 5 & 5 & 6,8 \\
VIH (\%) & 6,2 & 6 & 5,8 \\
\hline
\end{tabular}

Tabla 2. Servicios de Salud de Chile según tasas de tuberculosis en relación al promedio nacional. Año 2012

\begin{tabular}{|lcc|}
\hline & $\begin{array}{c}\text { Servicios de Salud } \\
\text { con tasas sobre } \\
\text { promedio nacional }\end{array}$ & $\begin{array}{c}\text { Servicios de Salud } \\
\text { con tasas bajo } \\
\text { promedio nacional }\end{array}$ \\
\hline n de servicios & 15 & 14 \\
$\begin{array}{l}\text { Promedio tasa } \\
\left(\mathrm{x} 10^{5}\right)\end{array}$ & 18 & 9,5 \\
$\begin{array}{l}\text { Rango tasas } \\
\left(\mathrm{x} 10^{5}\right)\end{array}$ & $13,8-32,2$ & $6-12,5$ \\
\hline
\end{tabular}

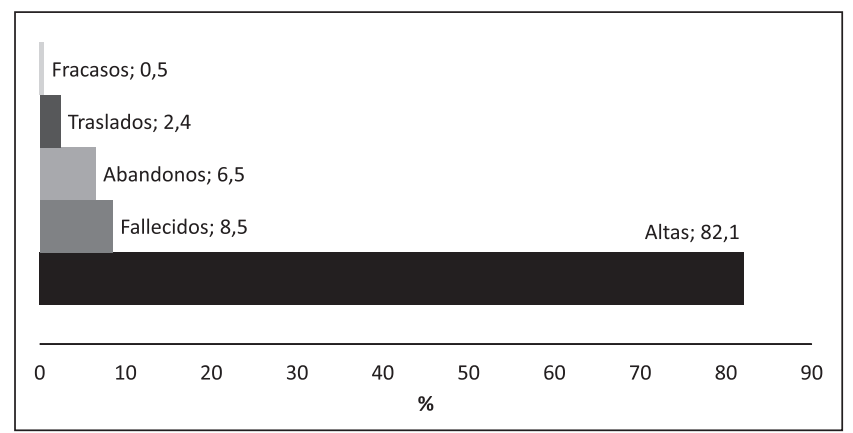

Figura 3. Resultados de terapia de tuberculosis cohorte de Tuberculosis pulmonar bacilífera en Chile. 2011.

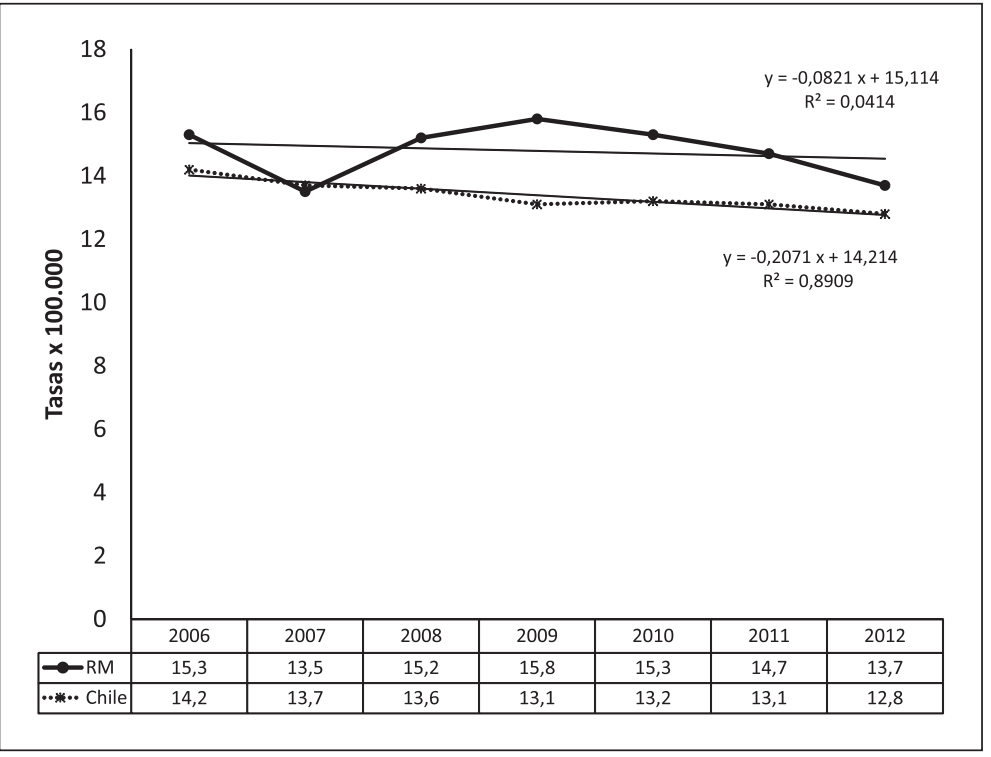

Figura 4. Incidencia de tuberculosis en la Región Metropolitana de Chile. Período 2006-2012. 
entre el año 2006 y 2012 muestra que 7058 personas adquirieron TBC (casi 3 casos diariamente) de los cuales 3.273 son pulmonares bacilíferos (más de un caso diario). En esta región el 67\% los Servicios de Salud muestran una incidencia sobre el promedio nacional, con una tasa promedio de $15,6 \times 100.000$ habitantes (Tabla 3).

El año 2012 los Servicios de Salud de la RM con mayores tasas son el Central, Sur, Norte y Occidente (Figura 5). En ese año se reportaron 393 pacientes

Tabla 3. Servicios de Salud de la Región Metropolitana de Chile según tasas de tuberculosis en relación al promedio nacional. Año 2012

\begin{tabular}{|lcc|}
\hline & $\begin{array}{c}\text { Servicios de Salud } \\
\text { con tasas sobre } \\
\text { promedio nacional }\end{array}$ & $\begin{array}{c}\text { Servicios de Salud } \\
\text { con tasas bajo } \\
\text { promedio nacional }\end{array}$ \\
\hline n de servicios & 4 & 2 \\
\hline $\begin{array}{l}\text { Promedio } \\
\text { tasa }\left(\mathrm{x} 10^{5}\right)\end{array}$ & 15,6 & 7,2 \\
$\begin{array}{l}\text { Rango } \\
\text { tasas }\left(\mathrm{x} 10^{5}\right)\end{array}$ & $14,6-16,6$ & $7,1-7,2$ \\
\hline
\end{tabular}

con TBC que pertenecían a alguno de los grupos de riesgo, siendo los más frecuentes el adulto mayor $(35,8 \%)$ y los migrantes $(20 \%)$, (Tabla 4).

La región tiene una población de adultos mayores de 650.285 habitantes, en los cuales se reportaron 169 casos de TBC, lo que representa una tasa de $25,9 \times 100.000$.

La pesquisa baciloscópica se ha mantenido estacionaria en la RM en el período 2008-2011, con un promedio anual de 90.082 baciloscopías, lo que significa que diariamente se realizan casi 62 baciloscopías (Figura 6).

Tabla 4. Proporción de grupos vulnerables en la incidencia de tuberculosis de la Región Metropolitana de Chile. Año 2012

\begin{tabular}{|lc|}
\hline Privados de libertad (\%) & 7,3 \\
\hline Migrantes (\%) & 20 \\
VIH (\%) & 11 \\
\hline
\end{tabular}

Figura 5. Tasas $x 10^{5}$ de incidencia tuberculosis todas las localizaciones y pulmonar bacilífera según Servicio de Salud de la Región Metropolitana. Chile, 2012.
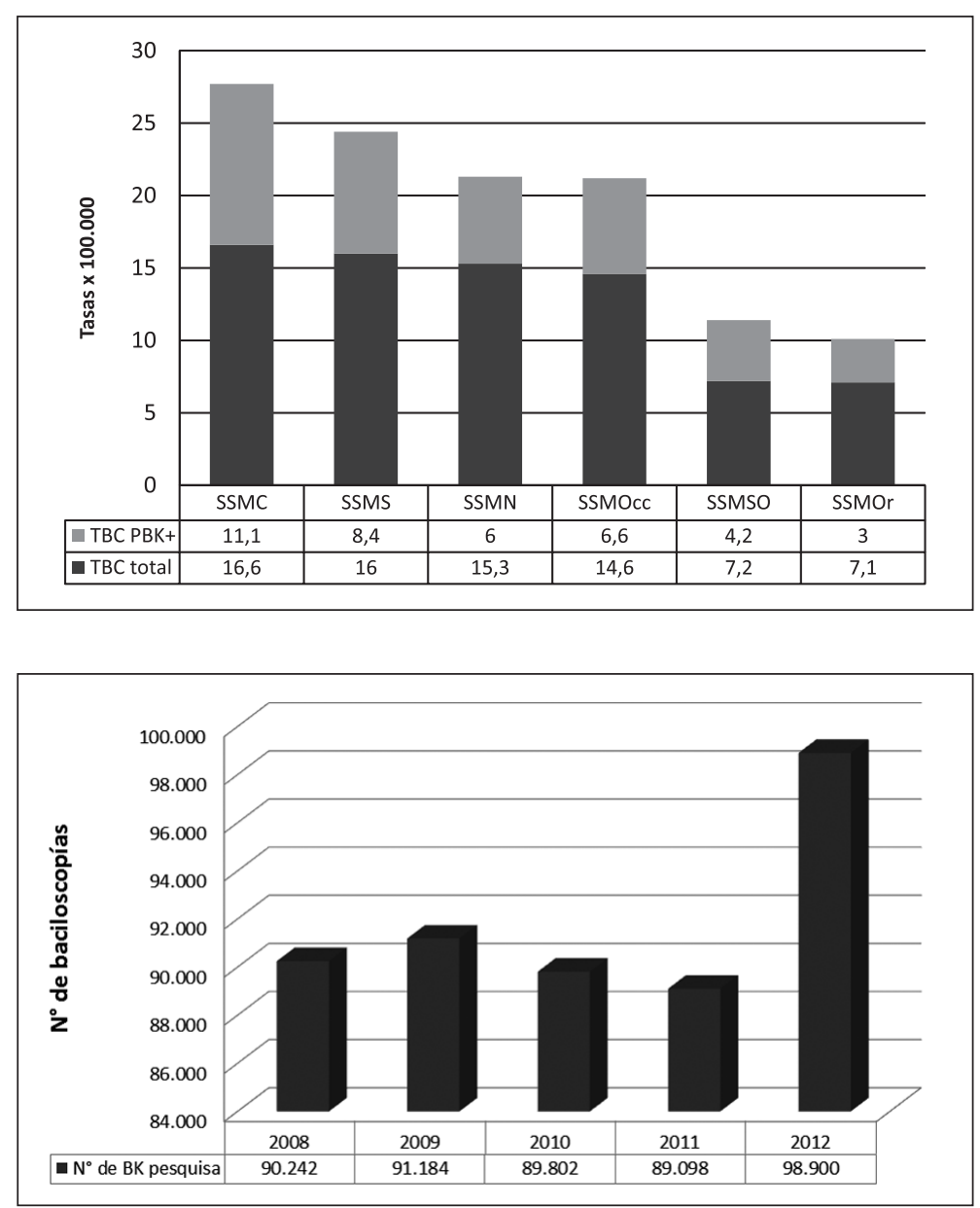

Figura 6. Número de baciloscopías (BK) de pesquisa en la Región Metropolitana. Período 2009-2012. 
En el año 2012 se logró aumentar las baciloscopías, llegando a 99.128 (incremento de 8.824 exámenes que corresponde a 9,8\% de aumento con respecto al año precedente). Los Servicios de Salud que incrementaron la pesquisa son el Central y Sur. 15 comunas $(28,9 \%)$ presentan la mayor incidencia ( $>20 \times 100.000$ habitantes), con una tasa de TBC muy superior al promedio nacional, ya que ésta alcanza a 29,34 x 100.000 habitantes (Tabla 5).

Datos preliminares indicarían que en la estratificación por comunas de la RM casi la mitad de la población se encontraría en una etapa avanzada de eliminación, con una tasa promedio de TBC de todas las localizaciones cercana a $6 \times 100.000$ habitantes (Tabla 6).

Los resultados del tratamiento de la cohorte de TBC pulmonar bacilífera virgen a terapias previas, con 2.257 casos acumulados entre el año 2007-2011, muestran un éxito terapéutico de $80,3 \%$, inferior a la meta OMS $(85 \%)$ y a la meta nacional $(90 \%)$, debido a la alta proporción de abandonos $(9,1 \%)$, que está distante de la meta deseada de menos de 5\% (Tabla 7). En la evaluación de la cohorte del año 2011 de la RM el Servicio de Salud con mayor éxito terapéutico, que es el Oriente, tampoco alcanza la meta nacional, ya que tiene un éxito de $88,9 \%$ (Tabla 8 y Figura 7).

\section{Áreas de intervención para lograr avances en el Control de la TBC}

Proponemos algunos ajustes estratégicos en diversas áreas de las intervenciones contra la $\mathrm{TBC}$, con el objetivo de contribuir a contener la endemia mediante la reducción de las fuentes de transmisión. Las siguientes son las medidas propuestas:

\section{Incorporar mejoras en el diagnóstico de los infectados y enfermos de TBC mediante}

a) Intensificación de la pesquisa baciloscópica en Atención Primaria hasta llegar a un índice de pesquisa de 50 (50 baciloscopías por cada 1.000 consultas de morbilidad general de adultos) y, además, intensificación de la pesquisa activa so-

Tabla 5. Comunas de la Región Metropolitana con tasas de incidencia de $\mathrm{TBC}>20 \times 100.000$ habitantes

\begin{tabular}{|lcc|}
\hline Comuna & n de casos & Tasa x 10 \\
Santiago & 104 & 65,0 \\
Recoleta & 47 & 38,5 \\
Lo Espejo & 32 & 32,9 \\
P. Aguirre Cerda & 29 & 32,0 \\
Cerro Navia & 37 & 28,1 \\
San Joaquin & 21 & 27,9 \\
Independencia & 13 & 26,0 \\
Lo Prado & 23 & 25,5 \\
Pudahuel & 70 & 25,5 \\
María Pinto & 3 & 25,4 \\
Melipilla & 27 & 25,1 \\
Estación Central & 26 & 23,7 \\
El Monte & 7 & 22,2 \\
\hline Cerrillos & 14 & 21,5 \\
\hline San José de Maipo & 3 & 20,8 \\
\hline
\end{tabular}

Tabla 6. Estratificación de las comunas de la Región Metropolitana según tasa de incidencia de Tuberculosis. Año 2012

\begin{tabular}{|ccccc|}
\hline $\begin{array}{c}\text { Promedio tasa } \mathbf{x} \mathbf{1 0}^{\mathbf{5}} \\
\text { TBC comunal }\end{array}$ & $\begin{array}{c}\text { Casos TBC } \\
\text { (n) }\end{array}$ & $\begin{array}{c}\text { Comunas } \\
\text { (n) }\end{array}$ & Población & $\begin{array}{c}\text { \% de población de la } \\
\text { Región Metropolitana }\end{array}$ \\
\hline 6 & 204 & 17 & 3.419 .067 & 48,8 \\
12,3 & 222 & 12 & 1.810 .446 & 25,8 \\
17,4 & 108 & 9 & 621.226 & 8,9 \\
29,3 & 456 & 15 & 1.162 .048 & 16,6 \\
\hline
\end{tabular}

Tabla 7. Eficacia del tratamiento de la tuberculosis en la Región Metropolitana y Chile

\begin{tabular}{|lccc|}
\hline & Éxito terapéutico & Meta OMS & Meta de Chile \\
\hline Región Metropolitana 2007-2011 & $80,3 \%$ & $85 \%$ & $90 \%$ \\
Chile 2011 & $82,1 \%$ & & \\
\hline
\end{tabular}


Tabla 8. Resultados de terapia de tuberculosis según Servicio de Salud de la Región Metropolitana. Año 2011

\begin{tabular}{|lccccc|}
\hline Servicio de Salud & $\begin{array}{c}\text { Altas } \\
\mathbf{\%}\end{array}$ & $\begin{array}{c}\text { Abandonos } \\
\mathbf{\%}\end{array}$ & $\begin{array}{c}\text { Fracasos } \\
\mathbf{\%}\end{array}$ & $\begin{array}{c}\text { Fallecidos } \\
\mathbf{\%}\end{array}$ & $\begin{array}{c}\text { Traslados } \\
\mathbf{\%}\end{array}$ \\
\hline Norte & 76,1 & 13,4 & 0,0 & 4,5 & 6,0 \\
Occidente & 87,9 & 1,5 & 0,0 & 12,1 & 0,0 \\
Central & 76,8 & 5,4 & 1,8 & 1,8 & 12,5 \\
Oriente & 88,9 & 0,0 & 0,0 & 8,3 & 2,8 \\
\hline Sur & 81,9 & 7,6 & 1,0 & 7,6 & 1,9 \\
Sur-Oriente & 88,3 & 2,9 & 1,0 & 5,8 & 1,9 \\
\hline
\end{tabular}

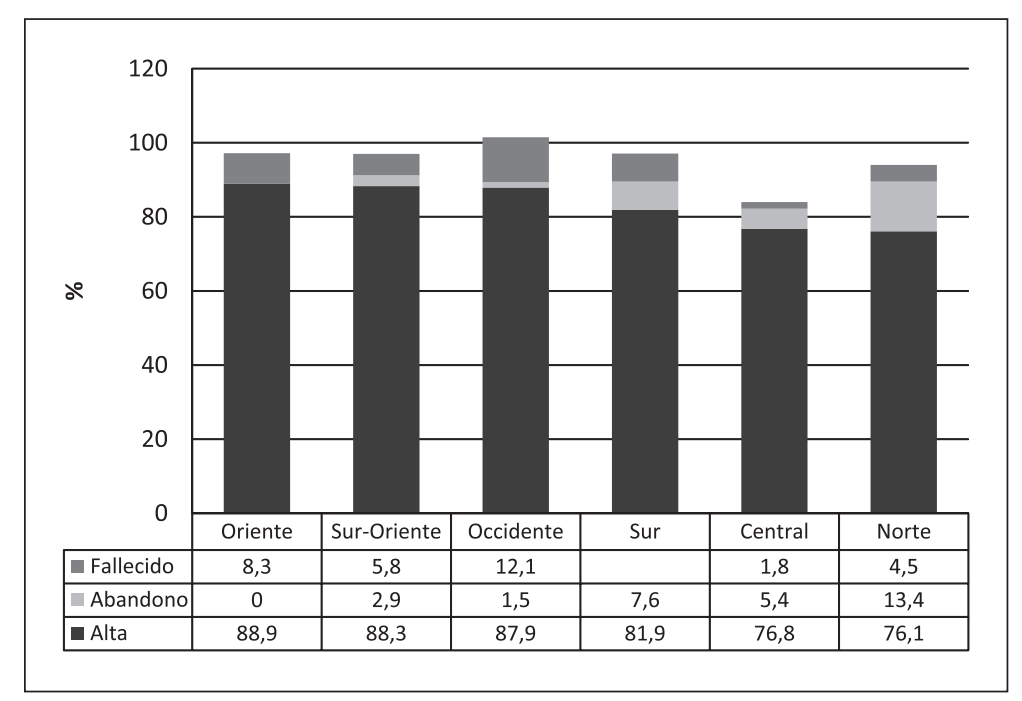

Figura 7. Resultados de terapia de tuberculosis cohorte pulmonar bacilíferos virgen a terapia previa según Servicio de Salud de la Región Metropolitana. Año 2011 bre población vulnerable como adultos mayores, privados de libertad (PPL), infectados por VIH, migrantes, diabéticos, sectores poblacionales de alta incidencia (comunas y unidades vecinales) y contactos intrafamiliares y de lugares de estudio o de trabajo. También intensificar la pesquisa en Salas ERA (Enfermedades Respiratorias del Adulto), realizando una baciloscopía semestral en su población cautiva y a todos los nuevos ingresos, exacerbaciones de patologías crónicas y neumonías.

b) Disponer de mejores técnicas de evaluación de la infección para el diagnóstico de TBC latente. En reemplazo o como complemento de la reacción de tuberculina (PPD) se están utilizando los llamados IGRAs (Interferon Gamma Release Assays), aunque aún no tienen cabida en el estado actual del Programa Nacional de Control de la TBC, debemos conocerlos y seguirlos utilizando a nivel individual.

Los IGRAs son técnicas que miden la liberación de interferón gama (INFy) por parte de linfocitos sensibilizados con antígenos del Mycobac- terium tuberculosis. Este ensayo se puede realizar in vitro con sangre periférica, de la cual se extraen los glóbulos blancos y se ponen en contacto con proteínas derivadas del bacilo tuberculoso (ESAT-6 o CFP-10), lo que provoca la liberación de interferón gama el cual se puede medir por ELISA (Quantiferón TB Gold) o visualizar como manchas dentro de los linfocitos teñidos, en una reacción antígeno-anticuerpo coloreada ( $T$-spot). La interpretación de los resultados de la prueba del QT-TB Gold dependerá de la relación de los niveles basales de INF- $\gamma$ frente a los obtenidos con la provocación de un agente inespecífico (mitógeno) (Tabla 9). La sensibilidad del test es de alrededor de 70\%. El T-spot tiene una sensibilidad mayor, cercana a $90 \%$ ya que detecta el INF- $\gamma$ dentro de linfocitos y cuantifica los puntos coloreados como spot o manchas aunque exista bajo recuento de estas células, mientras que el QT-TB Gold muestra resultados proporcionales al nivel de linfocitos sensibilizados. La especificidad de ambos métodos es de $99 \%$.

Los resultados de estos tests son menos pre- 
Tabla 9. Interpretación de resultados de Quantiferón TB Gold

\begin{tabular}{|lccc|}
\hline Interpretación & INF- $\gamma$ basal & $\begin{array}{c}\text { (INF- } \gamma \text { post exposición a ESAT-6 o } \\
\text { CFP-10)-(INF- } \gamma \text { basal) }\end{array}$ & $\begin{array}{c}\text { (INF- } \gamma \text { post exposición a } \\
\text { mitógeno)-(INF- } \gamma \text { basal) }\end{array}$ \\
Positivo & Cualquier & $\geq 0,35 \mathrm{UI} / \mathrm{ml} \mathrm{y} \geq 50 \%$ basal & Cualquier \\
Negativo & $\leq 0,7$ & $<0,35$ & $\geq 0,5$ \\
Indeterminado & $\leq 0,7$ & $<0,35$ & $<0,5$ \\
Indeterminado & $>0,7$ & $<50 \%$ basal & Cualquier \\
\hline
\end{tabular}

cisos en pacientes con infección por $\mathrm{VIH}$ con recuentos de $\mathrm{CD} 4$ inferiores a 100 céls $/ \mathrm{mL}$ y en enfermos que están utilizando quimioterapia antineoplásica. Han sido empleados para detectar infección tuberculosa en pacientes que recibirán terapia con Factor de Necrosis Tumoral alfa (TNF- $\alpha$ ) debido a enfermedades inmuno-reumatológicas como la artritis reumatoide. También en infectados VIH con CD4 mayor de $100 \mathrm{y}$ en trasplantados sometidos a terapia inmunosupresiva. Los IGRAs no ven afectados sus resultados por efecto de la vacunación BCG ni por la infección causada por micobacterias ambientales y pueden ser útiles cuando los valores del PPD no discriminan adecuadamente a infectados de no infectados por el M. tuberculosis. No requieren de lectores acreditados ni segundas visitas para medir los resultados.

c) Introducir métodos diagnósticos más sensibles y rápidos de la enfermedad. Existen técnicas de biología molecular que permiten, mediante sondas correspondientes a segmentos específicos del ADN de las micobacterias (IS6110), identificar en horas la existencia de un M. tuberculosis variedad humana. Incluso puede detectar la presencia de mutaciones en genes que codifican la resistencia a drogas (Tabla 10), permitiendo el diagnóstico de TB-MDR (TBC multiresistente) que deben recibir otros esquemas terapéuticos.

La OMS recientemente está propiciando uno de estos métodos de diagnóstico más rápidos, sensibles y específicos, el llamado gene Xpert TB/RIF ${ }^{6,7}$, basado en una de las técnicas de multiplicación genética derivadas de la reacción de la polimerasa en cadena (PCR), automatizada y de gran bioseguridad para el operador. Tiene una sensibilidad de $95 \%$ para detectar resistencia a rifampicina, por la presencia del gen mutado que codifica la resistencia a este fármaco (gen rpoB). Se propone para ser utilizado en grupos de riesgo de TB-MDR en pacientes antes tratados, PPL, VIH, migrantes, contactos de TB-MDR y en enfermos que no negativizan la baciloscopía al final del $2^{\circ}$ mes de tratamiento. Entre las grandes ven-
Tabla 10. Mutaciones genéticas de micobacteria tuberculosa asociadas a resistencia a fármacos contra la tuberculosis

\begin{tabular}{|lc|}
\hline Fármaco & $\begin{array}{c}\text { Mutación Gen } \\
\text { Isoniacida }\end{array}$ KatG, inhA, ahpC, kasA \\
Rifampicina & $r p o B$ \\
Pirazinamida & $p n c A$ \\
Etambutol & $e m b B$ \\
Estreptomicina & $r p s L, r r s$ \\
Fluorquinolonas & gyrA, gyrB \\
Etionamida & $i n h A$, etaAlethA \\
Cicloserina & alr $A$, Ddl \\
PAS & Desconocido \\
\hline
\end{tabular}

tajas del uso de este procedimiento se destacan: -reducción del tiempo de diagnóstico de la TBC a menos de $2 \mathrm{~h}$, -reducción del tiempo del estudio de susceptibilidad a fármacos anti-tuberculosos a menos de $2 \mathrm{~h}$ (versus los 75 días que demoran los estudios de sensibilidad fenotípicos habituales), y -reducción del tiempo de inicio de la terapia en pacientes con TBC y baciloscopías negativas a pocos días, frente a los 60 días que demoran los cultivos micobacterianos.

\section{Garantizar la oportunidad y eficacia del tratamiento preventivo y curativo de la TBC}

a) Tratamiento de la tuberculosis latente: $\mathrm{La}$ quimioprofilaxis (QMP) o tratamiento preventivo de la tuberculosis con fármacos específicos en infectados, PPD + , ha demostrado su utilidad en numerosos ensayos según la adherencia y tiempo de terapia útil ${ }^{8,9,10}$. En población norteamericana general se ha encontrado un riesgo de progresión de la TBC latente a enfermedad de 0,7 casos $/ 100$ personas/año. El riesgo de progresión a TBC activa es mayor en personas con infección por VIH (10\% anual) y en personas con viraje tuberculínico reciente (aumento de la induración 
del PPD de más de $6 \mathrm{~mm}$ en el plazo de 2 años). Los pacientes con infección por VIH y ausencia de TBC activa que tienen PPD $+>5 \mathrm{~mm}$ deben ser considerados candidatos para QMP, lo que reduce en $60 \%$ el riesgo de desarrollar una TBC activa. Otro grupo que se beneficia son los niños menores de 5 años que tuvieron contacto reciente con pacientes bacilíferos, lo que puede ser ampliado hasta menores de 15 años, cuando se decide intensificar los programas de QMP a nivel poblacional. Se utiliza isoniacida $5 \mathrm{mg} / \mathrm{kg} /$ día (habitualmente $300 \mathrm{mg}$ ) durante 9 meses. El empleo de otros fármacos por tiempos más breves mejora la adherencia, pero no ha demostrado mayor eficacia que la isoniacida sola y algunas asociaciones pueden ocasionar más reacciones $\operatorname{adversas}^{11}$.

b) Optimizar el tratamiento de la tuberculosis adaptado a las diferentes poblaciones bacilares (bacilos metabólicamente activos; con altos índices de crecimiento; bacilos con pulsos de actividad metabólica; bacilos intracelulares en medio ácido, y bacilos durmientes) $)^{12,13}$. Cada fármaco actúa preferentemente sobre una población bacilar específica (Figura 8).

Actualmente se están revisando las Normas del Programa Nacional de Control de la TBC, en las cuales se introducirán varios cambios en la terapia de la tuberculosis. Se reseñarán brevemente haciendo notar que aún se encuentran en etapa de aprobación por el Ministerio de Salud.

Se recomendará cambiar la segunda fase de tratamiento intermitente de bisemanal a trisemanal, lo que permite asegurar la biodisponibilidad del fármaco en situaciones de asistencia irregular (algunos pacientes no asisten regularmente a la administración de una dosis bisemanal, lo que en la práctica les significa estar una semana sin recibir la medicación). Además, la dosis de isoniacida trisemanal es menor que la bisemanal (600 mg contra $800 \mathrm{mg}$ por dosis), lo que reduciría la toxicidad dependiente de la dosis. Otros ajustes propuestos son la modificación de la dosis de etambutol de $1.200 \mathrm{mg}$ a $800 \mathrm{mg}$ en la fase diaria, ya que se ha demostrado la misma eficacia con la dosis más baja y con menor toxicidad ocular. Es también deseable disponer del estudio de susceptibilidad inicial en todos los pacientes, para realizar ajustes de la terapia más anticipados. Por ejemplo, se describe a nivel mundial un aumento de la resistencia a isoniacida y esto se debería detectar antes de pasar a la terapia intermitente con dos fármacos, la que corre el riesgo de inducir resistencia al fármaco acompañante (rifampicina) creando un caso de TB-MDR. Si se detecta resistencia a isoniacida se deberá mantener el etambutol durante toda la terapia. Asimismo se debe disponer del estudio de susceptibilidad para monitorizar la evolución de los casos con negativización tardía de la baciloscopía (Figura 9). En los pacientes con infección por VIH se recomienda prolongar la terapia intermitente a 7 meses, con una duración total de la terapia de 9 meses, para evitar las recaídas y asociar piridoxina, $50 \mathrm{mg}$ /día, para prevención de polineuropatía. La iniciación del tratamiento de la tuberculosis tiene preferencia en estos pacientes,
Figura 8. Actividad de fármacos antituberculosos en los tipos de población bacilar.

Figura 9. Monitorización de terapia mediante estudios de susceptibilidad a fármacos antituberculosos. BK=baciloscopía. $\mathrm{E}$ : Estreptomicina; $\mathrm{H}$ : isoniacida; R:rifampicina; Z: pirazinamida.
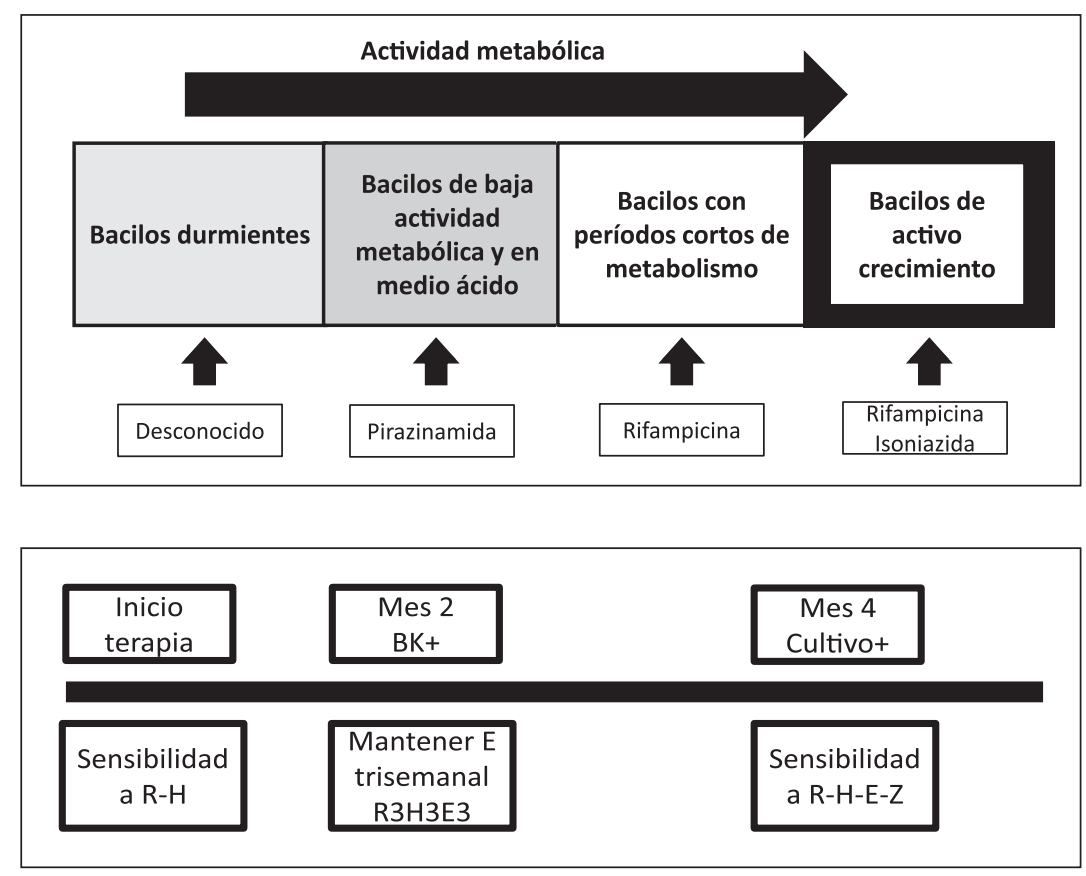
Tabla 11. Terapia diaria propuesta para tuberculosis multiresistente: TB-MDR

\begin{tabular}{|lcc|}
\hline Fármacos (mg) & $\begin{array}{r}\text { Fase inicial } \\
(\geq \mathbf{4} \text { meses) }\end{array}$ & $\begin{array}{c}\text { Fase continuidad } \\
(\geq \mathbf{8} \text { meses })\end{array}$ \\
\hline Kanamicina & 750 & - \\
Ethionamida & 750 & 750 \\
Moxifloxacino & 400 & 400 \\
Pirazinamida & 1.500 & 1.500 \\
Etambutol & 800 & 800 \\
\hline
\end{tabular}

pero la terapia antiretroviral (TAR) debe ser introducida o reanudada lo antes posible. En especial en aquellos con inmunodepresión severa, debe iniciarse en el más breve plazo (1-2 semanas de comenzado el tratamiento de la tuberculosis), lo que reduce la mortalidad. Si el CD4 es inferior a 50 debe monitorizarse la aparición del Síndrome de Reconstitución Inmune al asociar ambas terapias. Otra estrategia recomendable es utilizar las dosis fijas combinadas de medicamentos que reducen el número de comprimidos, con lo que mejora la adherencia a la terapia (reduce a 4 comprimidos la dosis diaria y la intermitente). Además, al estar combinados los fármacos, los pacientes los reciben asociados, previniendo la aparición de resistencia por mal ajuste de dosis o por selección de la ingesta de parte de la terapia ${ }^{14}$. Las dosis unitarias en cada comprimido de la fase diaria son: Isoniacida $75 \mathrm{mg}$, Rifampicina $150 \mathrm{mg}$, Etambutol 275 mg y Pirazinamida $400 \mathrm{mg}$ y cada comprimido de la fase intermitente (trisemanal) tiene Isoniacida $150 \mathrm{mg}$ y Rifampicina $150 \mathrm{mg}$.

c) Terapia de TB-MDR. Se debe garantizar una terapia adecuada a todos los enfermos multiresistentes con fármacos de segunda línea a los cuales sean sensibles. La eficacia debe ser monitorizada por la negativización de los cultivos. En nuestro país se utiliza una combinación que incluye nuevos fármacos (de segunda línea) como Ethionamida, Moxifloxacina y Kanamicina. Un esquema adecuado se presenta en la Tabla 11.

Se están ensayando nuevos medicamentos con actividad también sobre bacilos latentes. Por ejemplo, TMC 207 una Diarylquinolina que actúa sobre la ATP sintetasa de las micobacterias asociado a Rifapentina y Pirazinamida y OPC67683 que actúa sobre la síntesis de la pared de micobacteriana, asociado también a Rifampicina y Pirazinamida ${ }^{15,16}$

\section{Bibliografía}

1.- Global Tuberculosis Report 2012. WHO.

2.- Programa Nacional de Control de la Tuberculosis. Manual de Organización y Normas Técnicas. 2005. Chile.

3.- Evaluación del Programa de Control de la Tuberculosis 2012. PROCET. Ministerio de Salud de Chile.

4.- Evaluación Regional Metropolitana de Tuberculosis 2012. SEREMI. Ministerio de Salud de Chile.

5.- Updated Guidelines for Using Interferon Gamma Release Assays to Detect Mycobacterium tuberculosis Infection-United States, 2010. Morbidity and Mortality Weekly Report. June 25, 2010 / Vol. 59 / No RR-5.

6.- TUBERCULOSIS DIAGNOSTICS Xpert MTB/RIF Test. WHO RECOMMENDATIONS. February 2013. http://who.int/tb/laboratory/mtbrifrollout

7.- Update on Xpert MTB/RIF roll out Xpert MTB/RIF 2012. WHO. December 2012 www.stoptb.org/wg/gli/ xpert.asp

8.- COMSTOCK G W. Isoniazid prophylaxis among alaskan esquimos. Am Rev Resp Dis 1979; 119: 827-30.

9.- IUAT Committee on prophylaxis: Efficacy of various durations of isoniazid preventive therapy for tuberculosis: Five years of follow-up in the IUAT trial. Bull WHO 1982; 60: 555-64.

10.- COMSTOCK G W. How much isoniazid is needed for prophylaxis? Am Rev Resp Dis 1970; 101: 780-2.

11.- JASMER R M, SAUKKONEN J J, BLUMBERG H M, DALEY C L, BERNARDO J, VITTINGHOFF E, et al. Short-Course Rifampin and Pyrazinamide Compared with Isoniazid for Latent Tuberculosis Infection: A Multicenter Clinical Trial. Ann Intern Med 2002; 137: 640-7.

12.- HU Y, COATES A R, MITCHISON D A. Sterilising action of pyrazinamide in models of dormant and rifampicin-tolerant Mycobacterium tuberculosis Int $\mathrm{J}$ Tuberc Lung Dis 2006, 10: 317-22.

13.- MITCHISON D A. "The Diagnosis and Therapy of Tuberculosis During the Past 100 Years". Am J Respir Crit Care Med 2005, 171: 699-706.

14.- LIENHARDT C, COOK S V, BURGOS M, YORKEEDWARDS V, RIGOUTS L, ANYO G, et al. For the Study C Trial Group. Efficacy and Safety of a 4-Drug Fixed-Dose Combination Regimen Compared With Separate Drugs for Treatment of Pulmonary Tuberculosis. The Study C Randomized Controlled Trial. JAMA 2011; 305: 1415-23.

15.- BURMAN W J, VAN WINKLE R. Wakes Up: Development of Tuberculosis Treatment in the 21st Century. Clin Infect Dis 2010; 50 (S3): S165-S172.

16.- FARGA V, CAMINERO J A. Tuberculosis. Tercera Edición. Editorial Mediterráneo. Santiago de Chile. 2011.

Correspondencia a:

Dr. Carlos Peña M.

Email: carpemanti@hotmail.com 